University of South Carolina

Scholar Commons

$7-1-2001$

\title{
Low Frequency Noise in GaN Metal Semiconductor and Metal Oxide Semiconductor Field Effect Transistors
}

\author{
S. L. Rumyantsev \\ N. Pala \\ M. S. Shur \\ R. Gaska \\ M. E. Levinshtein
}

See next page for additional authors

Follow this and additional works at: https://scholarcommons.sc.edu/elct_facpub

Part of the Electrical and Electronics Commons, and the Other Electrical and Computer Engineering Commons

Publication Info

Published in Journal of Applied Physics, Volume 90, Issue 1, 2001, pages 310-314.

(C) Journal of Applied Physics 2001, American Institute of Physics (AIP).

Rumyantsev, S. L., Pala, N., Shur, M. S., Gaska, R., Levinshtein, M. E., Khan, M. A., Simin, G., Hu, X., \& Yang, J. (1 July 2001). Low Frequency Noise in GaN Metal Semiconductor and Metal Oxide Semiconductor Field Effect Transistors. Journal of Applied Physics, 90 (1), 310-314. http://dx.doi.org/10.1063/1.1372364

This Article is brought to you by the Electrical Engineering, Department of at Scholar Commons. It has been accepted for inclusion in Faculty Publications by an authorized administrator of Scholar Commons. For more information, please contact digres@mailbox.sc.edu. 


\section{Author(s)}

S. L. Rumyantsev, N. Pala, M. S. Shur, R. Gaska, M. E. Levinshtein, M. Asif Khan, Grigory Simin, X. Hu, and J. Yang 


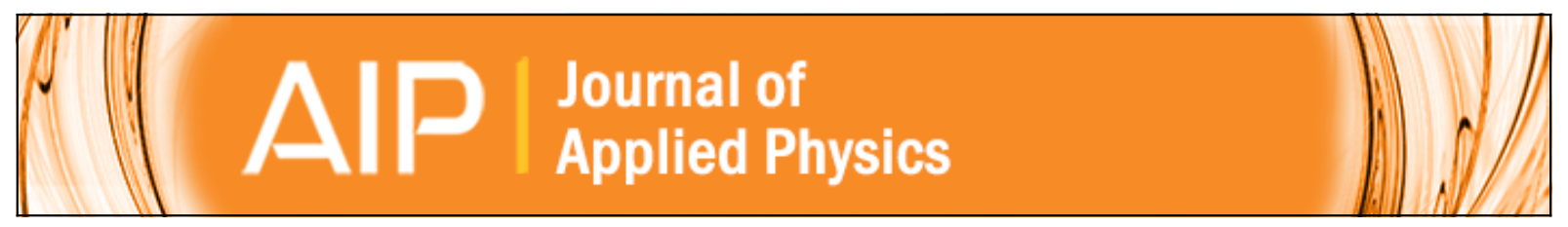

\section{Low frequency noise in GaN metal semiconductor and metal oxide semiconductor field effect transistors}

S. L. Rumyantsev, N. Pala, M. S. Shur, R. Gaska, M. E. Levinshtein, M. Asif Khan, G. Simin, X. Hu, and J. Yang

Citation: Journal of Applied Physics 90, 310 (2001); doi: 10.1063/1.1372364

View online: http://dx.doi.org/10.1063/1.1372364

View Table of Contents: http://scitation.aip.org/content/aip/journal/jap/90/1?ver=pdfcov

Published by the AIP Publishing

\section{Articles you may be interested in}

Low-frequency noise in AIN/AIGaN/GaN metal-insulator-semiconductor devices: A comparison with Schottky devices

J. Appl. Phys. 116, 054510 (2014); 10.1063/1.4892486

Carrier trapping and current collapse mechanism in GaN metal-semiconductor field-effect transistors

Appl. Phys. Lett. 84, 1970 (2004); 10.1063/1.1682700

Highly doped thin-channel GaN-metal-semiconductor field-effect transistors

Appl. Phys. Lett. 78, 769 (2001); 10.1063/1.1344577

On the origin of low frequency noise in GaAs metal-semiconductor field-effect transistors

J. Appl. Phys. 87, 517 (2000); 10.1063/1.371892

High-temperature reliability of GaN metal semiconductor field-effect transistor and bipolar junction transistor J. Appl. Phys. 85, 7931 (1999); 10.1063/1.370610

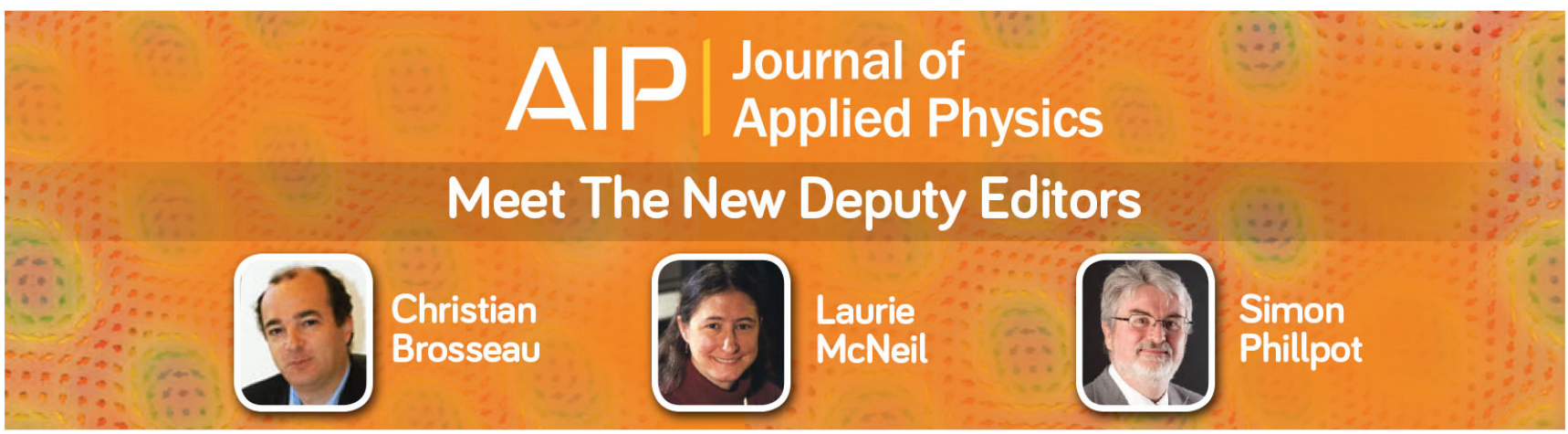




\title{
Low frequency noise in GaN metal semiconductor and metal oxide semiconductor field effect transistors
}

\author{
S. L. Rumyantsev, ${ }^{\text {a) }}$ N. Pala, ${ }^{\text {b) }}$ M. S. Shur, and R. Gaska ${ }^{\text {c) }}$ \\ Department of Electrical, Computer, and Systems Engineering and Center for Integrated Electronics and \\ Electronics Manufacturing, CII 9017, Rensselaer Polytechnic Institute, Troy, New York 12180-3590 \\ M. E. Levinshtein \\ Solid State Electronics Division, The Ioffe Physical-Technical Institute of Russian Academy of Sciences, \\ 194021, St. Petersburg, Russia
}

M. Asif Khan, G. Simin, X. Hu, and J. Yang

Department of Electrical and Computer Engineering, University of South Carolina, Columbia, South Carolina 29208

(Received 22 January 2001; accepted for publication 27 March 2001)

\begin{abstract}
The low frequency noise in GaN field effect transistors has been studied as function of drain and gate biases. The noise dependence on the gate bias points out to the bulk origin of the low frequency noise. The Hooge parameter is found to be around $2 \times 10^{-3}$ to $3 \times 10^{-3}$. Temperature dependence of the noise reveals a weak contribution of generation-recombination noise at elevated temperatures. (ㅇ 2001 American Institute of Physics. [DOI: 10.1063/1.1372364]
\end{abstract}

\section{INTRODUCTION}

A recent report on GaN highly doped metal semiconductor field effect transistors (HD-MESFETs) ${ }^{1}$ showed that these devices (especially short channel MESFETs) have a potential to compete with conventional $\mathrm{AlGaN} / \mathrm{GaN}$ heterostructure field effect transistors (HFETs). One of the most important parameters of the microwave transistors is the level of the low frequency noise, which determines the device suitability for microwave applications.

In this article, we present the experimental results on the bias and temperature dependence of the low frequency noise in HD-MESFETs and in GaN thin channel highly doped metal oxide semiconductor field effect transistors (HDMOSFETs). The analysis of the noise gate voltage dependence allows us to speculate about the noise sources location. The experimental results are compared with the noise data for bulk GaN and for AlGaN/GaN HEFTs.

\section{EXPERIMENTAL DETAILS}

The structures were grown by low-pressure metal organic chemical vapor deposition on (0001) sapphire substrates. The deposition of approximately $2 \mu \mathrm{m}$ of nominally undoped $\mathrm{GaN}$ was followed by the growth of a Si-doped $\mathrm{GaN}$ channel. The thickness and doping level of the channel (extracted from capacitance-voltage characteristics) were $\sim 60 \mathrm{~nm}$ and $10^{18} \mathrm{~cm}^{-3}$, respectively. The measured electron Hall mobility in the channel was close to $\mu=100 \mathrm{~cm}^{2} / \mathrm{V} \mathrm{s}$.

\footnotetext{
a) On leave from the Ioffe Institute of Russian Academy of Sciences, 194021 St-Petersburg.

${ }^{b)}$ Electronic mail: palan@rpi.edu

c)Also with Sensor Electronic Technology, Inc., 21 Cavalier Way, Latham, New York 12110
}

Prior to the HD-MOSFET fabrication, a $7 \mathrm{~nm} \mathrm{SiO}_{2}$ layer was deposited on a part of the heterostructures using plasma enhanced chemical vapor deposition.

The fabricated HD-MESFETs and HD-MOSFETs had the source-drain spacing of $4 \mu \mathrm{m}$ and the gate length of 1.5 $\mu \mathrm{m}$.

A low-frequency noise was measured in the frequency range from $1 \mathrm{~Hz}$ to $100 \mathrm{kHz}$ with the sources grounded. We used the probe station with the tungsten probes of $10 \mu \mathrm{m}$ diameter. A controlled pressure on the probes provided the contacts to the sample pads.

\section{RESULTS AND DISCUSSION}

The current-voltage characteristics of the HDMESFETs and HD-MOSFETs were similar and differed only in the threshold voltage, $V_{\text {th }}$, which was $V_{\text {th }}=(-4 \div$ $-5) \mathrm{V}$ and $V_{\text {th }}=(-7 \div-8) \mathrm{V}$ for HD-MESFETs and HDMOSFETs, respectively. Figure 1 shows the current-voltage characteristic of the HD-MESFET. The gate leakage current also shown in Fig. 1 did not exceed $I_{g}=10 \mathrm{nA}$ at drain bias $V_{d}=8 \mathrm{~V}$ and gate bias $V_{g}=5 \mathrm{~V}$ for both types of transistors. The measurements using transmission line model (TLM) structures showed that the contact resistance $R_{c}$ was negligible compared with the channel resistance.

The capacitance voltage measurements on the test structure with a relatively large area of the Schottky contact indicated that the doping profile of the GaN layer was uniform with doping density of $N_{d} \approx 10^{18} \mathrm{~cm}^{-3}$. The built-in voltage of the Schottky barrier was found to be $V_{\mathrm{bi}} \approx 1 \mathrm{~V}$.

The noise spectra of drain current fluctuations $S_{I d}$ had the form of $1 / f^{\Gamma}$ noise with $\Gamma$ close to unity $(\Gamma=1.0-1.15)$ for both HD-MESFETs and HD-MOSFETs. At low drain biases, $V_{d}<1 \mathrm{~V}$, the spectral noise density $S_{I d}$ was proportional to the square of the drain voltage $S_{I d} \sim V_{d}^{2}$, as expected. 


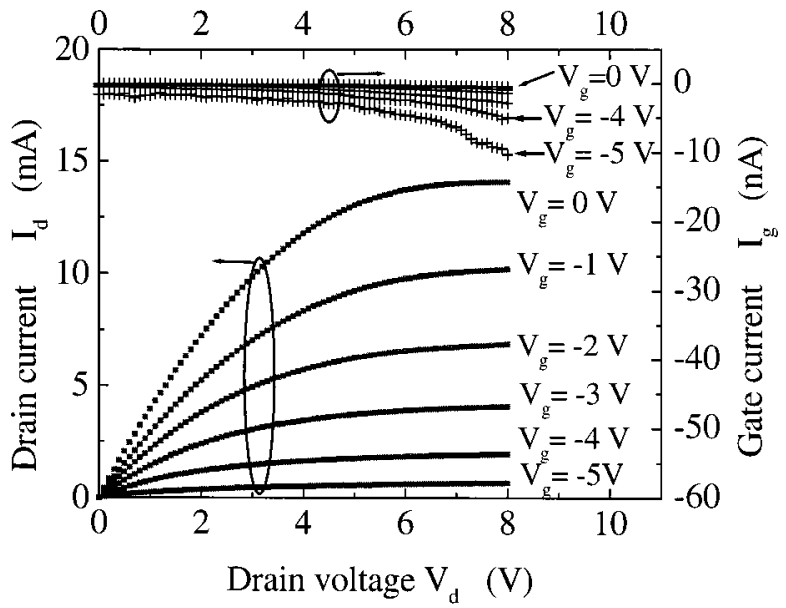

FIG. 1. Current-voltage characteristics of the MESFET under investigation. Crosses show the gate leakage current.

The noise temperature dependence revealed a weak contribution of one or two local levels. Figure 2 shows the temperature dependence of noise for one of the HD-MESFETs. As seen, the weak noise maxima shifted to higher temperatures with a frequency increase. This behavior is typical for the generation-recombination $(g-r)$ noise from a local level. ${ }^{2}$ However, the contribution of $g-r$ noise was too weak compared to the $1 / f$ noise in order to extract the local level parameters.

Many different noise sources in FETs might be important including the contribution of the gate leakage current, contact noise, bulk noise, surface noise, and the fluctuations of the Schottky barrier space charge region (SCR) width, $W$ (see Fig. 3). Depending on the device structure, different noise mechanisms are responsible for the main contribution to the overall noise. ${ }^{3-5}$ Since the observed low frequency noise was a superposition of the $1 / f$ and generationrecombination noise, we should analyze the possible location of the noise sources on the basis of both $1 / f$ and $g-r$ noise models.

\section{A. Contribution to noise from gate leakage current}

The gate leakage current in the MESFETs and MOSFETs under investigation did not exceed a few nanoamperes

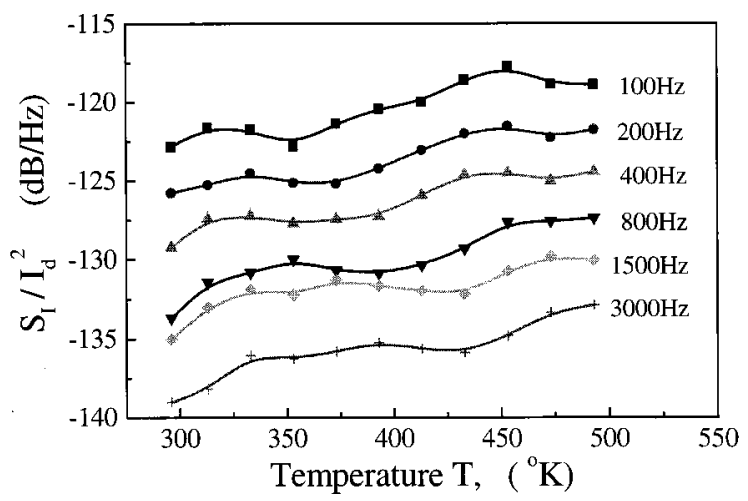

FIG. 2. Temperature dependence of noise $S_{I} / I_{d}^{2}$ for MESFET at different frequencies of analysis.

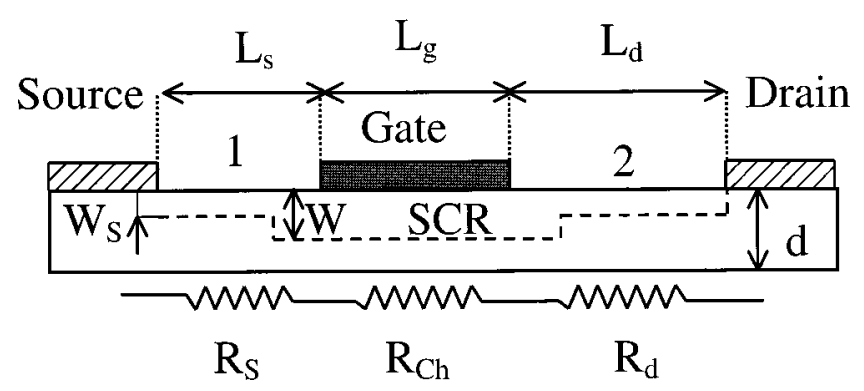

FIG. 3. The schematic view of the HD-MESFET. Also shown a simplified equivalent drain-to-source circuit, $R_{\mathrm{ds}}=R_{s}+R_{d}$.

in the linear regime of operation (see Fig. 1) and was 6-7 orders of magnitude smaller than the drain current. Hence, the gate leakage current should not contribute much to the output noise in these devices. ${ }^{6-8}$

\section{B. Contact contribution to noise}

In order to determine the contribution of the contact noise to the measured noise spectra, the noise measurements were performed on the TLM structures. Assuming that the contribution of the contact noise and of the noise from the $\mathrm{GaN}$ layer are not correlated and taking into account that the contact resistance is much smaller than the resistance of the $\mathrm{GaN}$ layer, the spectral noise density of the current fluctuations $S_{I} / I^{2}$ can be expressed as:

$$
\frac{S_{I}}{I^{2}}=\frac{S_{\mathrm{Rc}}+S_{\mathrm{GaN}}}{R_{\mathrm{GaN}}^{2}},
$$

where $S_{\mathrm{Rc}}$ and $S_{\mathrm{GaN}}$ are the spectral noise densities of the contact resistance and of the GaN layer resistance fluctuations, respectively, $R_{\mathrm{GaN}}$ is the resistance of the GaN layer between the pads of the TLM structure. In the limiting case when the contact noise dominant $\left(S_{\mathrm{Rc}} \gg S_{\mathrm{GaN}}\right)$, the spectral noise density, $S_{I} / I^{2}$, should be proportional to $L_{I}^{-2}$, where $L_{1}$ is the distance between the TLM contact pads:

$$
\frac{S_{I}}{I^{2}}=\frac{S_{\mathrm{Rc}}}{R_{\mathrm{GaN}}^{2}} \propto \frac{1}{L_{1}^{2}} .
$$

In the opposite limiting case, when $S_{\mathrm{Rc}} \ll S_{\mathrm{GaN}}$, the spectral noise density of the GaN layer resistance fluctuations is proportional to the reciprocal volume of the GaN layer (the bulk origin of the noise) or to the reciprocal $\mathrm{GaN}$ area (surface origin of the noise) between the contact pads. In both these cases:

$$
\frac{S_{I}}{I^{2}}=\frac{S_{\mathrm{GaN}}}{R_{\mathrm{GaN}}^{2}} \propto \frac{1}{L_{1}} .
$$

Figure 4 shows the dependence of the relative spectral noise density of the current fluctuations on the distance $L_{1}$ between the pads of the TLM structure. Since this dependence is close to the $1 / L_{1}$ law, we conclude that contacts do not contribute much to the overall noise. 


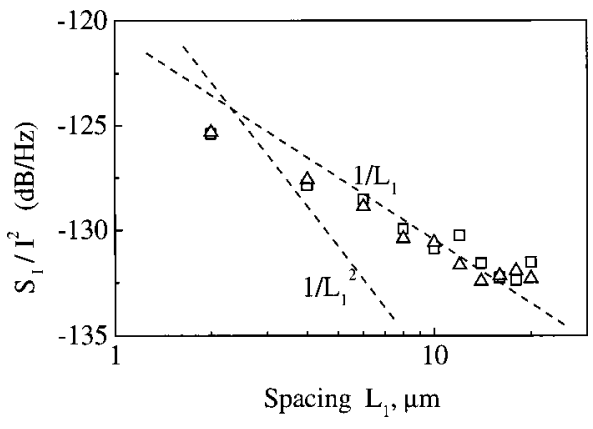

FIG. 4. The dependence of the relative spectral noise density $S_{I} / I^{2}$ on the distance $L_{I}$ between the pads of TLM structure. Frequency of analysis $f$ $=200 \mathrm{~Hz}$.

\section{Surface noise sources}

Let us first consider the location of the noise sources at the surface of $\mathrm{GaN}$ in source-gate and drain-gate regions. One of the possible mechanisms of the surface $g-r$ noise was analyzed in Ref. 9 (the $1 / f$ noise originated from the surface is often dominant in Si MOSFETs). ${ }^{10}$

For the noise sources located at the surface of regions 1 and 2 in Fig. 3, the relative spectral noise density of the short circuit drain current fluctuations can be presented in the following form:

$$
\frac{S_{I d}}{I_{d}^{2}}=\frac{S_{\mathrm{Rds}}}{R_{\mathrm{ds}}^{2}} \frac{R_{\mathrm{ds}}^{2}}{\left(R_{\mathrm{ds}}+R_{\mathrm{Ch}}\right)^{2}},
$$

where $R_{\mathrm{Ch}}$ is the channel resistance which depends on the gate voltage, $V_{g}, R_{\mathrm{ds}}=R_{d}+R_{s}$ is the resistance of sourcegate and drain-gate regions (regions 1 and 2 in Fig. 3), $S_{\mathrm{Rds}}$ is the spectral noise densities of the $R_{\mathrm{ds}}$ fluctuations. In this case, the noise gate voltage dependence is determined by the dependence of $R_{\mathrm{Ch}}$ on $V_{g}$.

The data points in Fig. 5 correspond to the experimental results for the dependence of noise on the drain current for HD-MESFETs and HD-MOSFETs at a constant drain bias. Within an experimental error, the noise behavior for both types of transistors was identical. This indicates that $\mathrm{SiO}_{2}$ film deposited in order to fabricate HD-MOSFETs does not

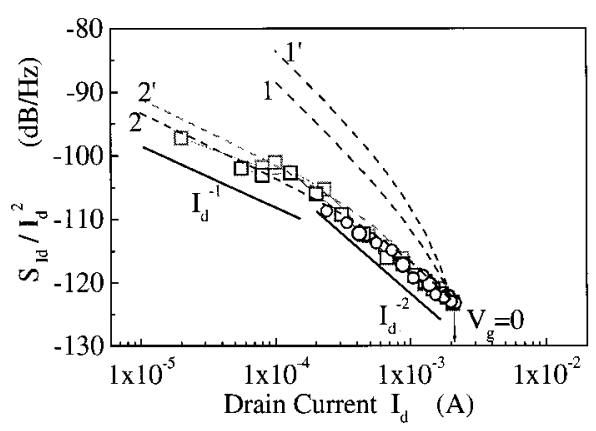

FIG. 5. The dependence of the of the relative spectral noise density of the drain current fluctuations on drain current. Drain voltage $V_{d}=0.5 \mathrm{~V}$. Frequency of analysis $f=200 \mathrm{~Hz}$. Different symbols show data for MESFETs and MOSFETs. Lines 1 and $1^{\prime}$ are calculated according to the Lauritzen model (see Ref. 11) [Eq. (10)] for $R_{\mathrm{ds}}=115 \Omega$ and $R_{\mathrm{ds}}=180 \Omega$, respectively. Lines 2 and $2^{\prime}$ are calculated according Eq. (14) for $R_{\mathrm{ds}}=115 \Omega$ and $R_{\mathrm{ds}}=180 \Omega$, respectively (bulk origin of noise). affect much the noise properties. As seen from Fig. 5, the relative spectral noise density $S_{I d} / I_{d}^{2}$ decreases with the drain current increase, i.e. with the gate voltage increase.

According to Eq. (4), noise $S_{I d} / I_{d}^{2}$ should decrease with the $R_{\mathrm{Ch}}$ increase, i.e., with the drain current decrease. Since the experimental dependence exhibits the opposite trend, the measured noise can not be explained by the surface noise.

\section{Fluctuations of the Schottky barrier SCR}

Another mechanism of a low frequency noise was analyzed by Lauritzen. ${ }^{11} \mathrm{He}$ assumed that the fluctuations of the charge state of the levels inside the depletion region of a $p-n$ junction or of a Schottky barrier result in the fluctuations of the depletion region width, $W$, and, consequently, in the fluctuations of the channel width and the channel resistance. For zero free carrier concentration in the depletion region and for a single time constant process contributing to noise, the expression for the equivalent gate voltage fluctuations $S_{V g}$ derived in Ref. 11 for the linear mode of operation is given by

$$
S_{V g}=A \frac{W^{3} \tau}{1+\omega^{2} \tau^{2}},
$$

where $A$ is the parameter which does not depend on gate voltage, $\omega=2 \pi f, f$ is the frequency, and $\tau$ is the fluctuation time constant. The $g-r$ noise of this origin was recently observed in GaAsFETs ${ }^{5}$ (the superposition of noise from several traps can result in the $1 / f$-like spectrum).

The spectral noise density of the channel resistance fluctuations is given by

$$
\frac{S_{\mathrm{RCh}}}{R_{\mathrm{Ch}}^{2}}=\frac{S_{V g} g^{2}}{I_{d}^{2}},
$$

where $g$ is the intrinsic transconductance. In the linear regime, the transconductance is inversely proportional to the depletion region width, $W$. Therefore, the dependence of the relative spectral noise density of the channel resistance fluctuations on the channel width $W$ can be expressed as:

$$
\frac{S_{\mathrm{RCh}}}{R_{\mathrm{Ch}}^{2}}=B \frac{W}{I_{d}^{2}} .
$$

The spectral noise density of the short circuit drain current fluctuations can be presented in the following form:

$$
\frac{S_{I d}}{I_{d}^{2}}=\frac{S_{\mathrm{Rch}}}{R_{\mathrm{Ch}}^{2}} \frac{R_{\mathrm{Ch}}^{2}}{\left(R_{\mathrm{ds}}+R_{\mathrm{ch}}\right)^{2}}=\frac{B W R_{\mathrm{Ch}}^{2}}{\left(R_{\mathrm{ds}}+R_{\mathrm{ch}}\right)^{2} I_{d}^{2}},
$$

where $B$ is the parameter which does not depend on the gate voltage.

In order to compare our experimental results (Fig. 5) with this model, the SCR width, $W$, should be expressed as a function of the drain current:

$$
W=\frac{\left(I_{\mathrm{fc}}-I_{d}\right) W_{0}}{\left(I_{\mathrm{fc}}-I_{d 0}\right)},
$$

where $I_{d 0}$ and $W_{0}$ are drain current and depletion region thickness, at $V_{g}=0$, respectively, $I_{\mathrm{fc}}$ is the full channel drain current. 
The substitution of Eq. (9) into Eq. (8) yields the dependence of noise on the drain current at the constant drain voltage:

$$
\frac{S_{I d}}{I_{d}^{2}}=B \frac{\left(I_{\mathrm{fc}}-I_{d}\right)\left(V_{d} / I_{d}-R_{\mathrm{ac}}\right)^{2} W_{0}}{\left(I_{\mathrm{fc}}-I_{d 0}\right) V_{d}^{2}} .
$$

In Fig. 5, lines 1 and $1^{\prime}$ are calculated using Eq. (10) for $R_{\mathrm{ds}}=115 \Omega$ and $R_{\mathrm{ds}}=180 \Omega$, respectively (see Appendix for $R_{\mathrm{ds}}$ estimates). In order to calculate the dependence of noise on the drain current, parameter $B$ was adjusted to fit the noise value at $V_{g}=0$. The thickness of the depletion region at $V_{g}$ $=0$ was taken to be $W_{0}=0.03 \mu \mathrm{m}$ (extracted from the capacitance-voltage measurements).

Figure 5 shows that this noise mechanism predicts a much faster increase of noise compared with that observed experimentally.

\section{E. Bulk location of the noise sources}

Van der Ziel ${ }^{12}$ assumed that fluctuations of the carrier density in the channel are responsible for the noise in FETs. Assuming that a single time constant process contributes to noise and neglecting the influence of resistance $R_{\mathrm{ds}}$ on noise, the spectral noise density of drain current fluctuations determined by this mechanisms are given by: ${ }^{12}$

$$
S_{I d}^{\prime}=\frac{4 I_{d} V_{d} q \mu \gamma \tau_{1}}{I_{g}^{2}\left(1+\omega^{2} \tau_{1}^{2}\right)},
$$

where $q$ is the electronic charge, $\mu$ is the electron mobility, $\gamma$ is constant, $L_{g}$ is the gate length, and $\tau_{1}$ is the fluctuation time constant.

For the linear regime of operation, Eq. (11) can be simplified:

$$
\frac{S_{I d}^{\prime}}{I_{d}^{2}}=\frac{S_{\mathrm{RCh}}}{R_{\mathrm{Ch}}^{2}}=\frac{4 \gamma \tau_{1}}{N_{\mathrm{Ch}}\left(1+\omega^{2} \tau_{1}^{2}\right)},
$$

where $N_{\mathrm{Ch}}$ is the number of electrons in the channel. A superposition of noise from several traps or from a continuous spectrum of levels results in the $1 / f$ like noise. ${ }^{13}$ Equation (12) takes into account only the noise sources located inside the channel. Assuming that the noise sources are not correlated and located both in the channel and in the lateral regions 1 and 2 (see Fig. 3), ${ }^{14,15}$ the spectral noise density of the drain current fluctuations can be presented in the following form:

$$
\frac{S_{I d}}{I_{d}^{2}}=\frac{S_{\mathrm{Rds}}}{R_{\mathrm{ds}}^{2}} \frac{R_{\mathrm{ds}}^{2}}{\left(R_{\mathrm{ds}}+R_{\mathrm{ch}}\right)^{2}}+\frac{S_{\mathrm{RCh}}}{R_{\mathrm{Ch}}^{2}} \frac{R_{\mathrm{Ch}}^{2}}{\left(R_{\mathrm{ds}}+R_{\mathrm{ch}}\right)^{2}} .
$$

In Eq. (12), $N_{\text {ch }}$ is the only parameter which depends on the gate voltage $V_{g}$. In the linear regime of operation, the number of electrons in the channel is inversely proportional to the channel resistance $\left(N_{\mathrm{ch}} \sim 1 / R_{\mathrm{ch}}\right)$. Hence, the expression for the spectral noise density of the drain current fluctuations is given by:

$$
\frac{S_{I d}}{I_{d}^{2}}=\frac{S_{\mathrm{Rds}}}{R_{\mathrm{ds}}^{2}} \frac{R_{\mathrm{ds}}^{2}}{\left(R_{\mathrm{ds}}+R_{\mathrm{ch}}\right)^{2}}+\frac{C R_{\mathrm{Ch}}^{3}}{\left(R_{\mathrm{ds}}+R_{\mathrm{ch}}\right)^{2}} .
$$

where $C$ is a parameter, which does not depend on gate voltage.

The bulk noise originated from the regions 1 and 2 in Fig. 3 should be of the same nature as the channel bulk noise. Therefore the spectral noise density $S_{\mathrm{Rds}} / R_{\mathrm{ds}}^{2}$ is given by

$$
\frac{S_{\mathrm{Rds}}}{R_{\mathrm{ds}}^{2}}=\frac{\Phi_{\mathrm{Ch} 0}}{\Phi_{\mathrm{ds}}}\left(\frac{S_{\mathrm{RCh}}}{R_{\mathrm{Ch}}^{2}}\right)_{V_{g}=0},
$$

where $\Phi_{\mathrm{Ch} 0}=\left(d-W_{0}\right) L_{g} Z$, is the channel volume at $V_{g}$ $=0, d$ is the full channel thickness, $Z$ is the channel width, and $\Phi_{\mathrm{ds}}$ is the volume of GaN layer between source-gate and gate-drain intervals (regions 1 and 2 in Fig. 3). We estimated $\Phi_{\mathrm{ds}}$ as $\Phi_{\mathrm{ds}}=\left(d-W_{0}\right)\left(L_{d}+L_{s}\right) Z$ for $R_{\mathrm{ds}}=180 \Omega$ and $\Phi_{\mathrm{ds}}$ $=d\left(L_{d}+L_{s}\right) Z$ for $R_{\mathrm{ds}}=115 \Omega$ (see Appendix for $R_{\mathrm{ds}}$ estimates).

Curves 2 and $2^{\prime}$ in Fig. 5 are calculated using Eq. (14) for $R_{\mathrm{ds}}=115 \Omega$ and $R_{\mathrm{ds}}=180 \Omega$, respectively. As can be seen from Fig. 5, the experimental data can be fitted quite well using the bulk noise model. At a low drain current, $\left(S_{I d} / I_{d}^{2}\right) \sim I_{d}^{-1}$. This reflects the fact that the noise depends on the channel volume as $1 / \Phi_{\mathrm{Ch}}$ [see Eq. (14)]. This is a usual behavior of the bulk $1 / f$ noise. At high drain currents and small gate voltages, the dependence of noise on drain current is close to $\left(S_{I d} / I_{d}^{2}\right) \sim I_{d}^{-2}$.

Since it appears that the noise sources in the transistors under investigation are located in the bulk of GaN layer, the Hooge parameter $\alpha=\left(S_{I} / I^{2}\right) N f$ ( $N$ is the total number of carriers in the sample) can be used to characterize the noise level. ${ }^{16}$ We estimated $\alpha=(2-3) \times 10^{-3}$ for the entire range of the gate voltages. These values of $\alpha$ are at least one order of magnitude smaller than those reported for bulk $\mathrm{GaN}^{17}$ and 3-5 orders of magnitude smaller than that recently reported for $p$-type GaN. ${ }^{18}$ The $\alpha$ values for HD-MESFETs and HDMOSFETs found in the present article are comparable with $\alpha$ values for $\mathrm{AlGaN} / \mathrm{GaN}$ HFETs. ${ }^{6,19,20}$

\section{CONCLUSION}

The measurements of the low frequency noise on $\mathrm{GaN}$ HD-MESFETs and HD-MOSFETs showed that the noise properties of MESFETs and MOSFETs are identical and that the drain and source contacts do not contribute much to the low frequency noise. The dependence of the noise on gate voltage indicates that the noise originates from the bulk of $\mathrm{GaN}$ in the channel and in the source to gate and drain to gate regions. We estimated the Hooge parameter $\alpha=(2-3)$ $\times 10^{-3}$. This value is about one order of magnitude smaller than the value of $\alpha$ reported for bulk $n$-type GaN. The temperature dependence of noise shows a weak contribution of $g-r$ noise at elevated temperatures.

\section{ACKNOWLEDGMENTS}

The work at RPI was supported by Office of Naval Research, Project monitor was Dr. J. Zolper. The work at USC was supported by the Ballistic Missile Defense Organization (BMDO) under Army SSDC Contract No. DASG60-98-10004, monitored by Dr. Brian Strickland and Dr. Kepi Wu. 
The work at SET, Inc. was supported by BMDO under SBIR program and monitored by WPAFB (Monitor Dr. F. Schuermeyer). One of the authors (M.E.L.) gratefully acknowledges support of European Research Office of the US Army under Contract No. 68171-99-M-6802.

\section{APPENDIX}

From Fig. 3, we find

$$
R_{\mathrm{ds}}=R_{d}+R_{s}=\frac{R_{0}}{1+\frac{L_{g}\left(d-W_{s}\right)}{\left(L_{d}+L_{s}\right)\left(d-W_{0}\right)}},
$$

where $R_{0} \approx 250 \Omega$ and $W_{0}=0.03 \mu \mathrm{m}$ are the output resistance and depletion region thickness $W$ at $V_{g}=0$. This equation yields $R_{\mathrm{ds}} \approx 115 \Omega$ for $W_{s}=0$ and $R_{\mathrm{ds}} \approx 160 \Omega$ for $W_{s}$ $=W \approx 0.03 \mu \mathrm{m}$.

Resistance $R_{\mathrm{ds}}$ can also be estimated from the transistor $\mathrm{dc}$ characteristics. The gate voltage dependence of the output resistance $R_{\text {out }}$ is given by (see Ref. 21, for example):

$$
R_{\mathrm{out}}=\frac{R_{0}}{1-\sqrt{\frac{V_{\mathrm{bi}}-V_{g}}{V_{\mathrm{po}}}}}+R_{\mathrm{ds}},
$$

where $V_{\text {po }}$ is the pinch-off voltage.

The intercept of the dependence of $R_{\text {out }}$ on

$$
\left(1-\sqrt{\frac{V_{\mathrm{bi}}-V_{g}}{V_{\mathrm{po}}}}\right)^{-1}
$$

should yield the value of $R_{\mathrm{ds}}$. The accuracy of this procedure is limited, since this technique is very sensitive to the values of $V_{\mathrm{bi}}$ and $V_{\mathrm{po}}$. This method yields $R_{\mathrm{ds}} \approx 115-180 \Omega$.
${ }^{1}$ R. Gaska, M. S. Shur, X. Hu, A. Khan, J. W. Yang, A. Taraki, G. Simin, J. Deng, T. Werner, S. Rumyantsev, and N. Pala, Appl. Phys. Lett. 78, 769 (2001).

${ }^{2}$ M. E. Levinshtein and S. L. Rumyantsev, Semicond. Sci. Technol. 9, 1183 (1994).

${ }^{3}$ J. Graffeuil, K. Tantrarongroj, and J. F. Sautereau, Solid-State Electron. 25, 367 (1982).

${ }^{4}$ M. Chertouk and A. Chovet, IEEE Trans. Electron Devices 43, 123 (1996).

${ }^{5}$ L. Dobrzanski and Z. Wolosiak, J. Appl. Phys. 87, 517 (2000).

${ }^{6}$ S. Rumyantsev, M. E. Levinshtein, R. Gaska, M. S. Shur, J. W. Jang, and M. A. Khan, J. Appl. Phys. 87, 1849 (2000).

${ }^{7}$ S. Rumyantsev, N. Pala, M. S. Shur, R. Gaska, M. E. Levinshtein, A. Khan, G. Simin, X. Hu, and J. W. Jang, J. Appl. Phys. 88, 6726 (2000).

${ }^{8}$ S. L. Rumyantsev, N. Pala, M. S. Shur, M. E. Levinshtein, R. Gaska, X. Hu, J. Yang, G. Simin, and M. Asif Khan, International Workshop on Nitride Semiconductors, 24-27, September 2000 Nagoya, Japan.

${ }^{9}$ P. A. Ivanov, M. E. Levinshtein, J. W. Palmour, and S. L. Rumyantsev, Semicond. Sci. Technol. 15, 164 (2000).

${ }^{10}$ J. H. Scofield and D. M. Fleetwood, IEEE Trans. Nucl. Sci. 38, 1567 (1991).

${ }^{11}$ P. O. Lauritzen, Solid-State Electron. 8, 41 (1965).

${ }^{12}$ A. van der Ziel, Proc. IEEE 51, 1670 (1965).

${ }^{13}$ N. V. Dyakonova, M. E. Levinshtein, S. L. Rumyantsev, Sov. Phys. Semicond. 25, 1241 (1991).

${ }^{14}$ J.-M. Peransin, P. Vignaud, D. Rigaud and L. K. J. Vandamme, IEEE Trans. Electron Devices 37, 2250 (1990).

${ }^{15}$ A. Balandin, Electron. Lett. 36, 912 (2000).

${ }^{16}$ F. N. Hooge, IEEE Trans. Electron Devices 41, 1926 (1994).

${ }^{17}$ M. E. Levinshtein, S. L. Rumyantsev, D. C. Look, R. J. Molnar, M. Asif Khan, G. Simin, V. Adivarahan, and M. S. Shur, J. Appl. Phys. 86, 5075 (1999).

${ }^{18}$ A. K. Rice and K. J. Malloy, J. Appl. Phys. 87, 7892 (2000).

${ }^{19}$ A. Balandin, S. V. Morozov, S. Cai, R. Li, K. L. Wang, G. Wijerathe, and C. R. Viswanathan, IEEE Trans. Microwave Theory Tech. 47, 1413 (1999).

${ }^{20}$ J. A. Garrido, B. E. Foutz, J. A. Smart, J. R. Shealy, M. J. Murphy, W. J. Schaff, and L. F. Eastman, Appl. Phys. Lett. 76, 3442 (2000).

${ }^{21}$ M. S. Shur, GaAs Devices and Circuits (Plenum, New York, 1987). 